\title{
Les essais du groupe marémoteur expérimental de Saint-Malo
}

\section{The tests on the experimental Saint-Malo unit}

\author{
PAR R. DUBOIS
}

CHEF DE LA DIVISION 《ESSAIS EXTÉRIEURS》

DES ÉTUDES ET REGHERCHES A ÉLECTRICITÉ DE FRANCE

\begin{abstract}
Présentation des méthodes et moyens mis en cuvre pour les essais de rendement et les essais en orifice du groupe marémoteur expérimental de Saint-Malo. Analyse des caractéristiques de l'écoulement dans le conduit hydraulique et des conditions particulières dues à la variation rapide des plans d'ean de part et d'autre de la machine.

Présentation des diagrammes tópographiques des rendements de la machine en pompe et en turbine. Analyse du comportement de la machine en orifice et présentation des caractéristiques de fonctionnement.
\end{abstract}

\begin{abstract}
Description of the methods and equipment used to measure the overall efficiency of the unit and test it under straight-through flow conditions. Analysis of flow characteristics in the hydraulic passage, and of special conditions resulting from rapid upstream and downstream water level variations.

Overall efficiency plots for the machine operating as a pump and as a turbine. Analysis of the behaviour of the machine under straightthrough flow conditions; presentation of the operating characteristics.
\end{abstract}

\section{I. - INTRODUCTION}

Après les réalisations des groupes bulbe aval de Cambeyrac et d'Argentat, et du groupe de Beaumont-Monteux, la possibilité d'installer «à la mer », à Säint-Malo, un groupe de caractéristiques plus voisines de celles des futurs groupes de la Rance, a été mise à profit pour poursuivre la mise au point du groupe bulbe marémoteur.

Depuis sa mise en service, fin 1959 , le groupe expérimental de Saint-Malo a fait l'objet d'essais très nombreux dans des domaines aussi divers que l'hydraulique, l'électro-mécanique ou la corrosion.

Le présent exposé ne traite pas de l'ensemble de ces essais, mais se limite aux mesures de rendement et aux essais en orifice dont le Département «Essais» des Etudes et Recherches Hydrauliques de l'Electricité de France a assuré l'exécution en collaboration avec le Constructeur.

- On ne reviendra pas ici sur les caractéristiques de l'aménagement de Saint-Malo, renvoyant pour cela à l'article de M. Kammerlocher dans la Revue Générale d'Electricité de mai 1960, mais on passera directement à l'exposé des essais, dont les têtes de chapitre sont :

- Description des installations de mesure;

- Caractéristiques de l'écoulement dans les deux sections de jaugeage;

- Influence sur les mesures des variations de niveau au cours d'un essai;

-- Présentation des résultats des essais de rendement;

- Essais en orifice. 


\section{II. - DESCRIPTION DES INSTALLATIONS POUR LA MESURE DU RENDEMENT GLOBAL DU GROUPE}

La figure 1 présente l'implantation générale de l'usine et des principales installations de mesure décrites ci-après.

1. Mesure du Débit (fig. 2).
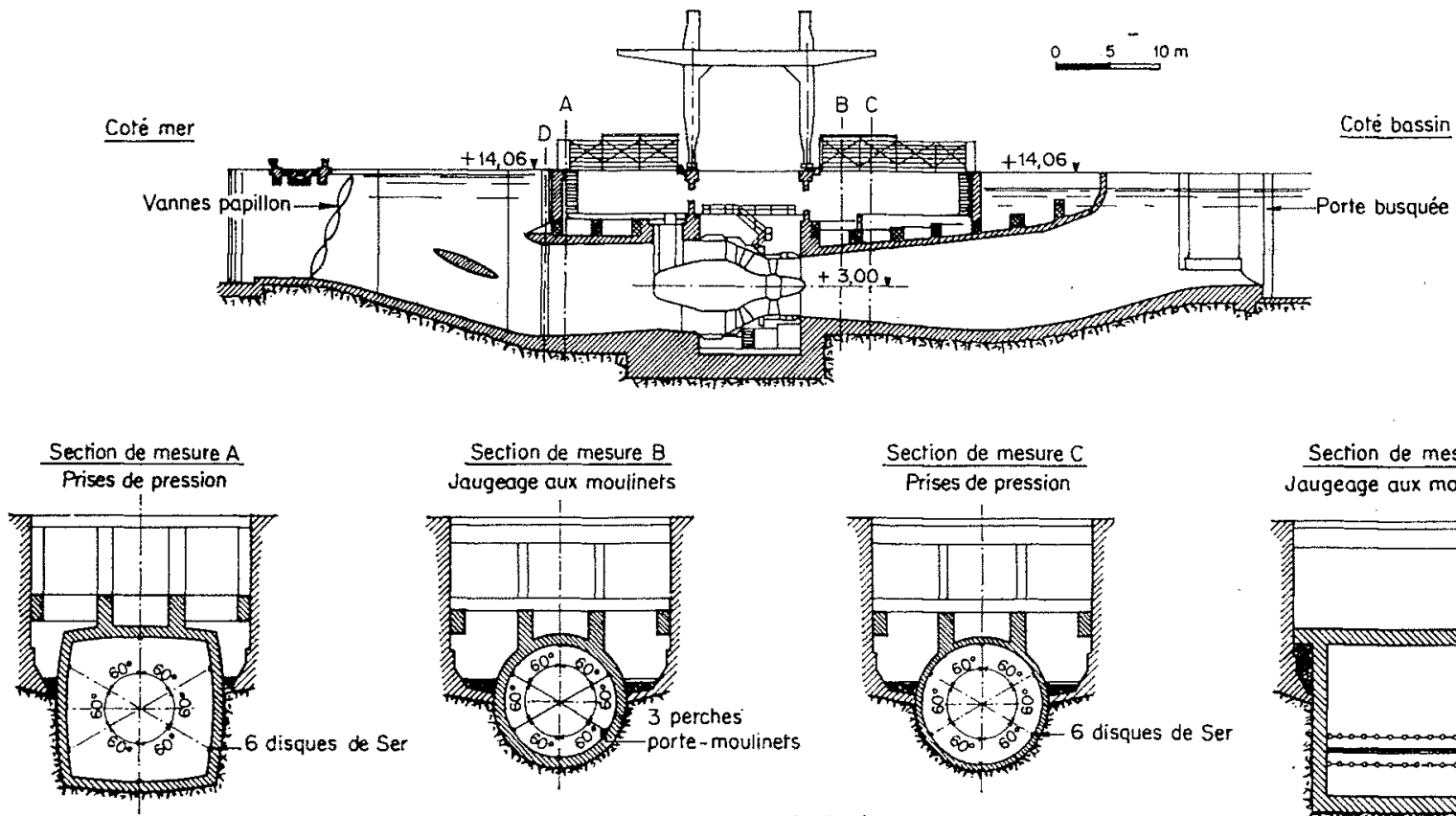

Section de mesure $C$ Prises de pression

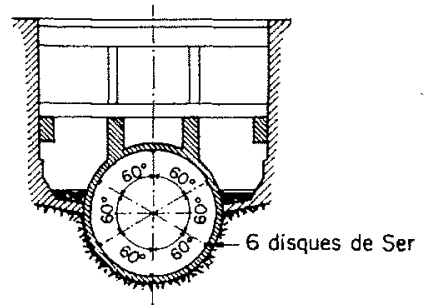

$0 \quad 2 \quad 6 \mathrm{~m}$
Section de mesure 0 Jaugeage aux moulinets

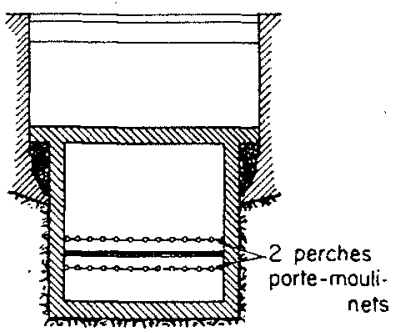

Fig. 1. - Schéma de l'installation. Coupes longitudinale et transversale.

Section de jaugeage $\mathrm{B}$ Repartition des points de mesure
Section de jaugeage 0

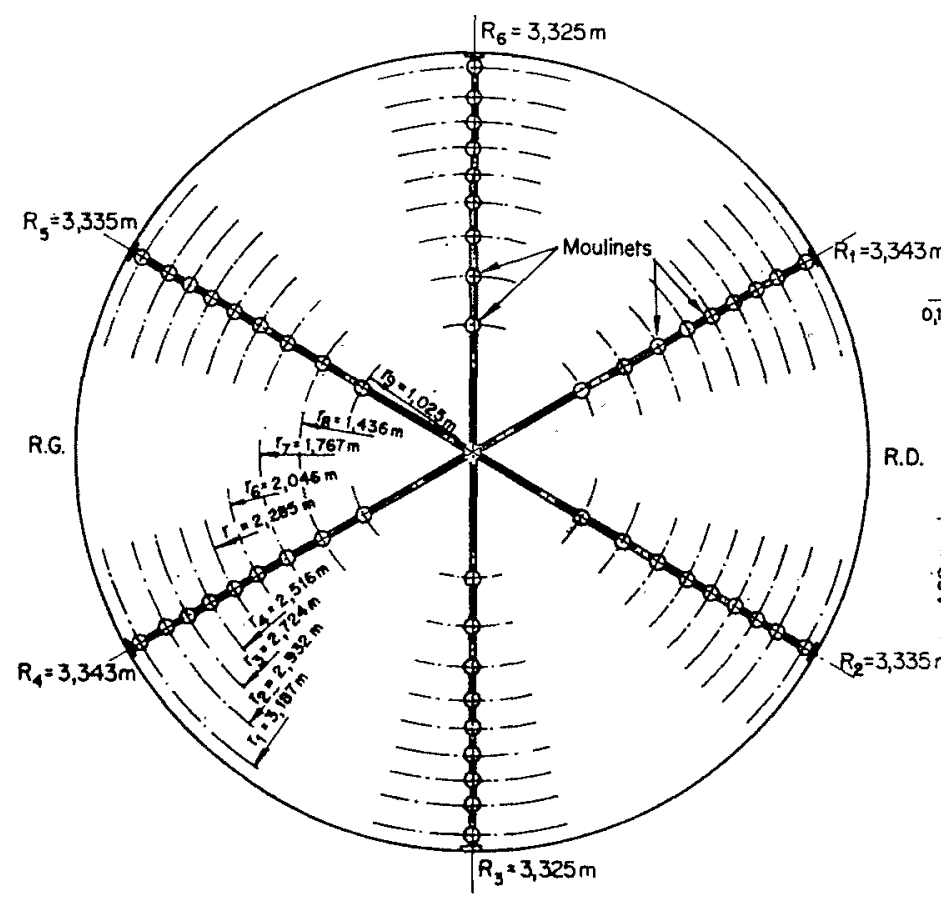

Répartition des verticales de mesure

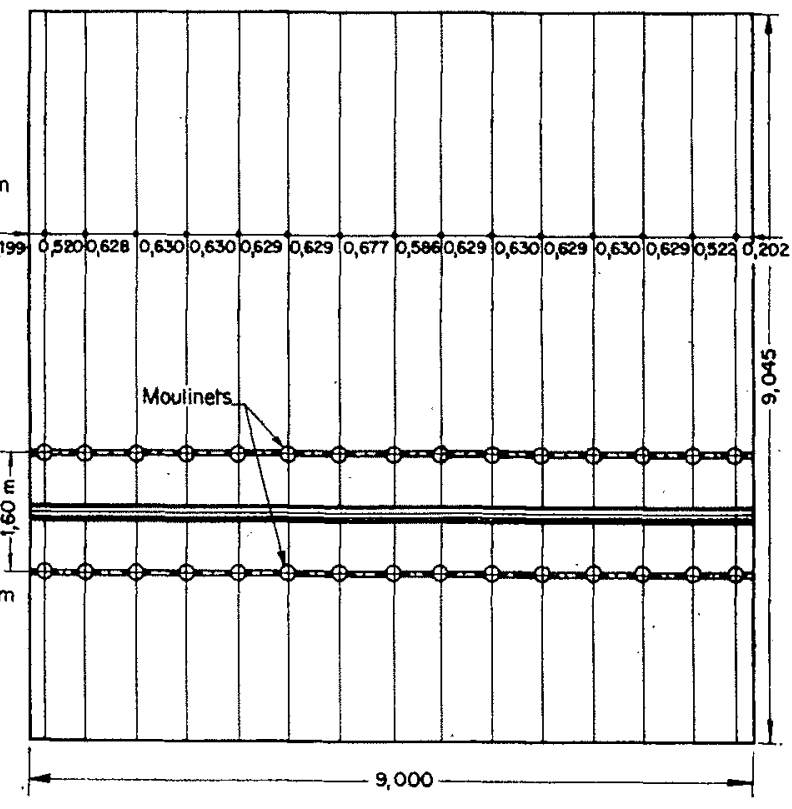

FIG. 2. - Définition des sections de jaugeage. 
Un dispositif différent a dû être adopté pour chacun des deux sens d'écoulement de l'eau.

Lorsque l'écoulement a lieu du bassin vers la mer on utilise dans la section $B$ un croisillon de 3 perches permettant la mesure de la vitesse ponctuelle en 55 points disposés sur 6 rayons décalés de 60 degrés.

Lorsque l'écoulement a lieu de la mer vers le bassin on utilise dans la section $D$ un cadre comportant deux perches horizontales supportant chacune quinze moulinets. Ce cadre se déplace de façon continue à la vitesse de $2,5 \mathrm{~cm} / \mathrm{s}$ de manière à explorer toute la section de jaugeage : les moulinets intègrent les vitesses ponctuelles rencontrées sur toute la hauteur du pertuis.

\section{Mesure de la chute.}

La chute nette et la hauteur d'élévation nette lors des fonctionnements en pompe ont été définies comme la différence d'altitude de la ligne de charge entre deux sections de mesure situées de part et d'autre de la machine, à l'intérieur du conduit hydraulique (sections $\mathrm{A}$ et $\mathrm{C}$ ). Ces sections ont été choisies pour permettre une mesure aussi correcte que possible dans les deux sens d'écoulement. Chaque section est équipée de six prises de pression statiques en disques de SER reliée chacune à un tube manométrique. L'écart assez faible entre les niveaux piézométriques extrêmes a permis, en utilisant simplement une contrepression obtenue à l'aide d'une colonne d'eau de mer, de mesurer l'altitude de la ligne piézométrique de façon très directe sans passer par l'intermédiaire d'un liquide manométrique.

\section{Mesure de la PUissance Électrique.}

La puissance électrique est mesurée de façon classique par la méthode des deux wattmètres avec des transformateurs, des wattmètres et des compteurs de classe 0,2 .

\section{III. - CARACTERISTIQUES DE L'ECOULEMENT DANS LES DEUX SECTIONS DE MESURE}

La difficulté majeure des mesures de rendement dans les aménagements de basse chute réside dans la mesure du débit, car la faible longueur du conduit hydraulique ne permet pas en général l'établissement d'un écoulement très régulier. Il est alors nécessaire d'analyser l'écoulement dans la section de jaugeage avant d'adopter une méthode de calcul du débit à partir des relevés de vitesses.

On peut faire sur l'écoulement dans le conduit hydraulique de Saint-Malo les remarques suivantes :

\section{SECTION DE JAUGEAGE côté BASSIN.}

Du fait de la forte convergence de l'écoulement entre l'entrée et la section de jaugeage, les caractéristiques de l'écoulement dans cette section se sont révélées satisfaisantes.

La répartition des vitesses se maintient sensiblement constante quel que soit le débit: le rapport des vitesses ponctuelles à une vitesse de référence se conserve à $\pm 1,7 \%$ près environ.

Le profil des vitesses moyennes par cercle en fonction du rayon présente l'allure très aplatie, caractéristique des ouvrages d'amenée courts.

La figure 3 représentant les vitesses relatives moyennes sur 60 essais, montre que, sur le cercle de mesure le plus proche de la paroi corres-

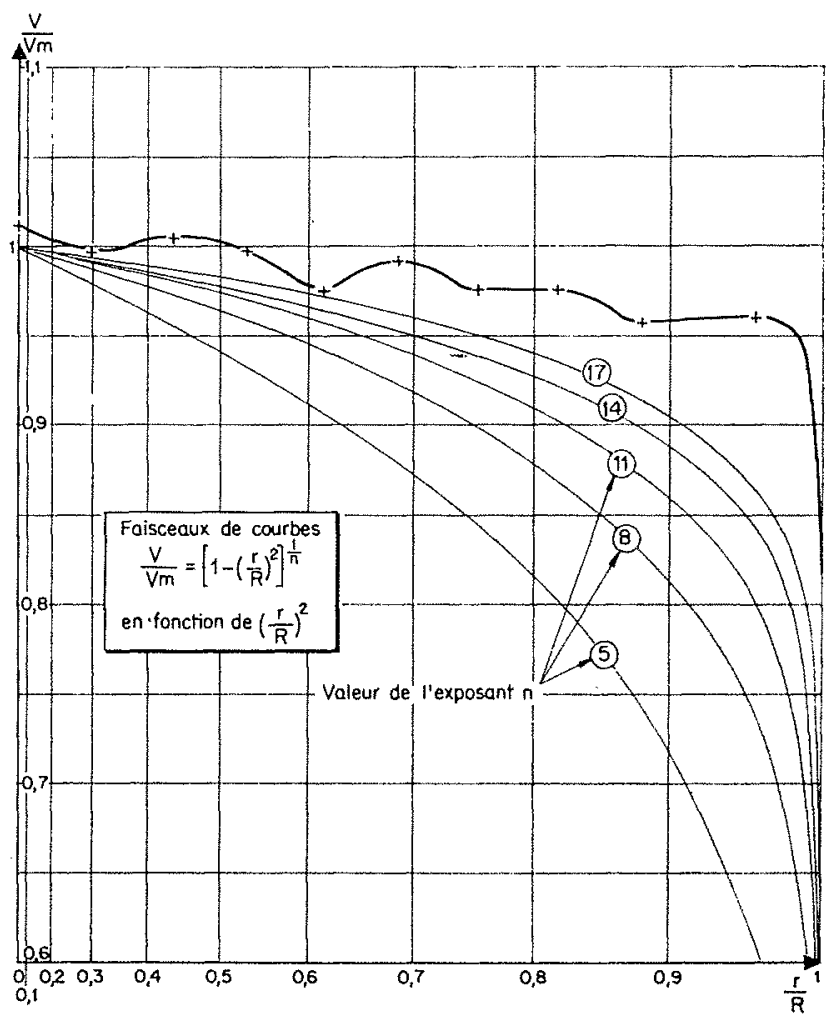

Fig. 3

Saint-Malo.

Répartition des vitesses dans la section de jaugeage Côté bassin. 
pondant à $(r / \mathrm{R})=0,955$, Ia vitesse est encore égale à $95 \%$ de sa valeur au centre, alors que pour une conduite de longueur notable, le profil des vitesses est en général à l'intérieur du faisceau de courbes représenté paramétré par l'exposant $n$.

On a par ailleurs constaté que l'écoulement était sensiblement de révolution, les profils des vitesses sur chacun des six rayons explorés différant au maximum de $\pm 2 \%$.

\section{SEction DE JAUGEAgE Côté MER.}

Les conditions d'alimentation de la section de jaugeage côté mer sont peu favorables, du fait de la proximité de l'avant-port où règnent à certaines heures de la marée de notables courants traversiers.

Le buton et son pilier support ont une influence favorable sur l'orientation des vitesses mais ils créent un sillage encore sensible dans la section de jaugeage.

On a donc été conduit à analyser l'écoulement par stationnement du cadre sur dix horizontales réparties sur la hauteur du pertuis. Cette exploration des vitesses ponctuelles a permis les remarques suivantes :

- Dans la partie inférieure du pertuis, les vitesses le long d'une horizontale varient peu; leur diminution est brutale au voisinage des parois.
- Dans la partie supérieure, les vitesses sur la rive Saint-Servan sont nettement supérieures à celles de la rive Saint-Malo. Ce phénomène, vraisemblablement dù à la dissymétrie de l'alímentation dans l'avant-port, est atténué dans la partie inférieure du pertuis par le pilier vertical du buton qui redresse l'écoulement.

Le long d'une verticale, près de la rive Saint-Malo, on observe une diminution sensible de vitesse vers une hauteur de 6 mètres, correspondant au sillage du buton; les vitesses n'y atteignent que $60 \%$ environ des valeurs relevées plus bas ou plus haut sur la même verticale.

- Près de la rive Saint-Servan, ce creux n'apparaît plus, masqué par la sensible augmentation des vitesses qui, dans le tiers supérieur du pertuis atteignent jusqu'à $130 \%$ de la valeur noyenne dans la partie inférieure.

On a cependant observé que, malgré cette dissymétrie notable de l'écoulement, la répartition des vitesses dans la section de jaugeage se conservait de façon acceptable, ce qui a permis de maintenir la méthode de jaugeage par intégration continue, en utilisant les essais par stationnement pour passer de la vitesse moyenne par verticale au débit. Dans ces conditions, l'erreur sur la mesure du débit dans le sens d'écoulement mer-bassin peut être évaluée à $2 \%$.

\section{IV. - INFLUENCE SUR LES MESURES DE LA VARIATION DES NIVEAUX AMONT ET AVAL AU COURS D'UN ESSAI}

La mesure précise du débit, que l'écoulement ait lieu dans un sens ou dans l'autre, a nécessité, en général, une durée d'essai de 10 minutes.

Pendant ces 10 minutes, du fait de la marée d'une part et de l'évolution du niveau du bassin d'autre part, la chute brute varie aux bornes de la machine. Le planning des essais a visé à limiter ces variations dans la mesure du possible en opérant au voisinage de la pleine mer et en faisant varier les niveaux amont et aval dans le même sens, mais il reste que, pour certains essais, la variation de chute a été notable, de l'ordre de 40 centimètres.

Le débit mesuré étant nécessairement le débit arithmétique moyen pendant l'essai, nous avons adoplé également pour la chute et la puissance la moyenne arithmétique des valeurs relevées à intervalle régulier pendant l'essai, sous réserve de vérifier que le point ainsi défini en puissance, débit et chute $\grave{a}$ un point de fonctionnement effectif de la machine.

Pour cela considérons dans le plan $Q, H$ les

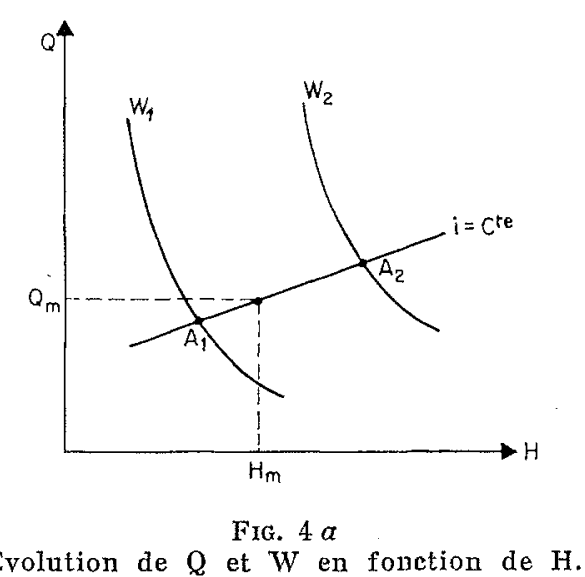


caractéristiques de fonctionnement de la machine définies par les courbes d'égale puissance et d'égale inclinaison des pales (fig. 4a) : évolution de $Q$ et de $W$ en fonction de $H$ ).

Au cours d'un essai l'inclinaison des pales est fixe; on se déplace donc, pendant cet essai, d'un point $A_{1}$ à un point $A_{2}$ sur une seule courbe $i=$ Cte. Or ces courbes, sur les turbines bulbe ou Kaplan en général, sont pratiquement des droites. Il en résulte, puisque $Q_{m}$ est le débit arithmétique moyen d'un essai, qu'il est nécessaire d'adopter de même pour $\mathbf{H}$ la valeur $\mathbf{H}_{m}$, moyenne arithmétique de la valeur de la chute au cours de l'essai.

Par contre, l'évolution de la puissance entre les points $A_{1}$ et $A_{2}$ n'est pas rigoureusement linéaire avec la chute.

Nous allons maintenant considérer l'évolution des grandeurs $Q, H$ et $W$ au cours d'un essai (fig. $4 b$ : évolution de W, Q, $H$ en fonction du temps).

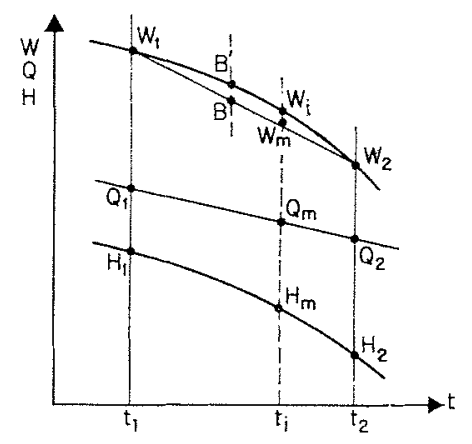

FiG. $4 b$

Evolution de W, Q, $\mathrm{H}$ en fonction du temps.

En ne faisant aucune hypothèse sur "la forme de variation de $Q$ et de $\ddot{H}$ au cours de l'essai, on a vu que les valeurs $H_{m}$ et $Q_{m}$ se correspondent, donc qu'elles ont lieu à l'instant $t_{i}$ entre $t_{1}$ et $t_{2}$. De même la puissance évolue entre $W_{1}$ et $\mathrm{W}_{2}$.

A l'instant $t_{i}$ la puissance effective est $W_{i}$, correspondant à $\mathrm{Q}_{m}$ et $\mathrm{H}_{m}$. Or la puissance qu'on prend en compte est $W_{m}$, moyenne arithmétique des puissances entre $W_{1}$ et $W_{2}$. On fait donc une erreur $\left(W_{i}-\mathbf{W}_{m}\right) / \mathbf{W}_{i}$.

On peut facilement évaluer une valeur par excès de l'écart $W_{i}-W_{m}$ d'un essai en prenant pour cette valeur le segment $\mathrm{BB}^{\prime}$ maximal entre $W_{1}$ et $W_{2}$. La valeur relative maximale du seg ment $\mathrm{BB}^{\prime}$ dans les essais les plus critiques n'a pas excédé $0,3 \%$.

En fait les variations de chute en fonction du temps sont sensiblement linéaires dans un intervalle de 10 minutes et, compte tenu de la très faible courbure des courbes $\mathrm{W}=f(\mathrm{H})$, l'écart $\mathrm{W}_{i} \longrightarrow \mathrm{W}_{m}$ est bien inférieur au segment $\mathrm{BB}^{\prime}$ maximal.

A titre d'exemple on a représenté sur la figure $4 c$ les variations de $Q, H$ et $W$ au cours d'un essai choisi parmi les plus critiques. La chute a varié de façon pratiquement linéaire de $40 \mathrm{~cm}$, le débit ayant parallèlement diminué de $2,32 \mathrm{~m}^{3} / \mathrm{s}$. La puissance au cours de l'essai est passée de 4315 à $4857 \mathrm{~kW}$. Cependant les valeurs de $W_{i}=4597$ et $W_{n}=4592$ ne diffèrent que de $0,1 / 100$.

Des vérifications analogues ont été faites pour différents essais comportant de notables variations de chute et, l'erreur commise ne dépassant pas $0,1 / 100$, on a négligé dans tous les cas de corriger la valeur obtenue $W_{n}$ de la puissance.

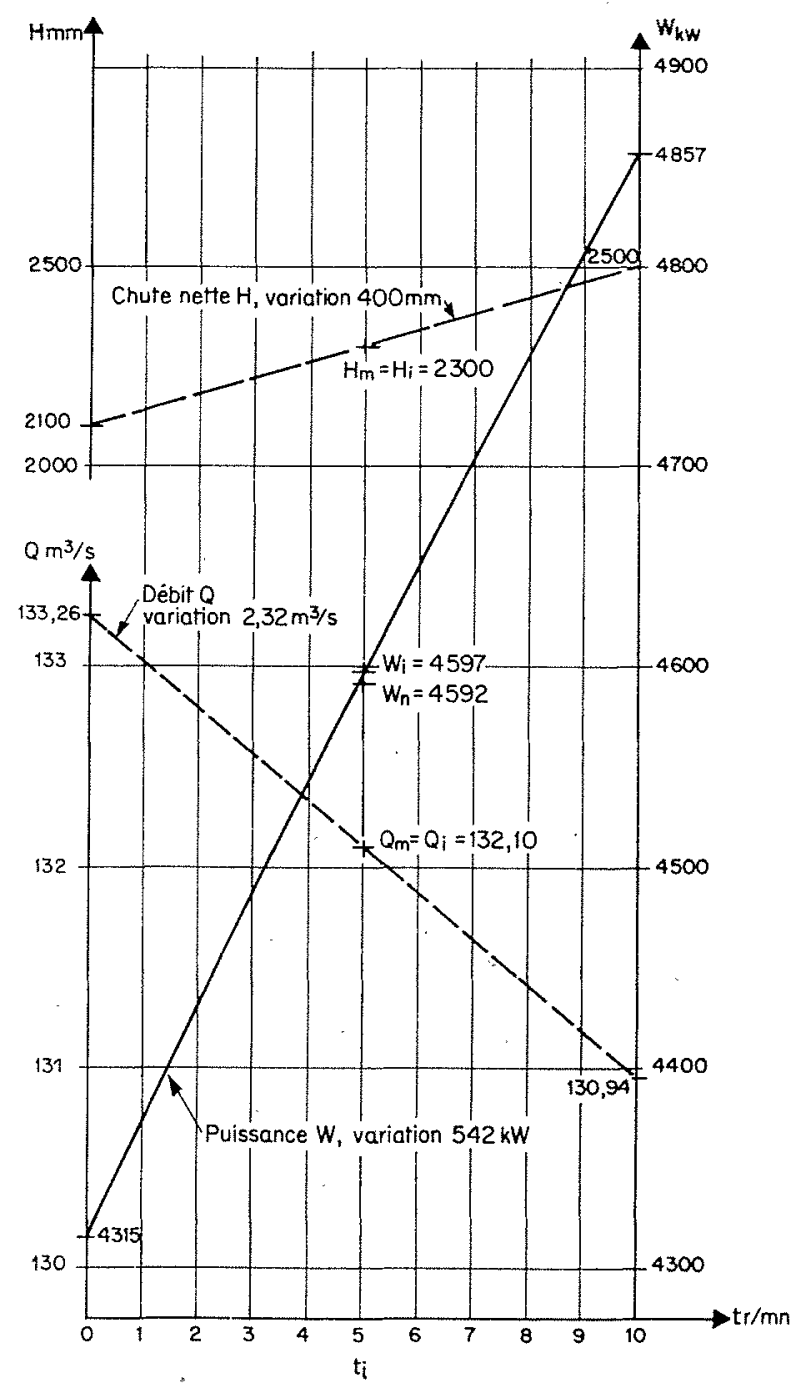

FIG. $4 c$

Variations de $\mathrm{H}, \mathrm{O}, \mathrm{W}$ au cours d'un essai de 10 minutes en pompe inversée.

(Essai X 4, décembre 1959; $i=12,6 ; \alpha=106$.) 


\section{V. - RESULTATS DES ESSAIS DE RENDEMENT}

Il convient tout d'abord de signaler que, de par son implantation et les sujétions d'exploitation du port, le domaine de fonctionnement $d u$ groupe de Saint-Malo est beaucoup plus limité que celui des futurs groupes de la Rance. La méthode utilisée pour extrapoler nos résultats d'essais à un domaine plus vaste fait dans ce même numéro l'objet de l'exposé de M. Chevalier.

Les diagrammes topographiques des rendements dans le plan $Q, H$ (fig. 5) donnent une représentation globale des performances de la machine. On a figuré en traits pleins les zones d'essais industriels à Saint-Malo, et en tirets l'extrapolation de ces résultats au domaine d'exploitation normal de la Rance.
Les rendements au cour de la colline pour les différents fonctionnements s'élèvent respectivement à :

- $75 \%$ en turbine inversée,

- $66 \%$ en pompe directe,

- $74 \%$ en pompe inversée.

Fn turbine directe la plage couverte par les essais est trop éloignée du cœur pour que l'extrapolation puisse être conduite avec assez de certitude.

Ces résultats trouvent surtout leur intérêt dans la comparaison des rendements du prototype avec ceux du modèle réduit dont traite également l'exposé de M. Chevalier.

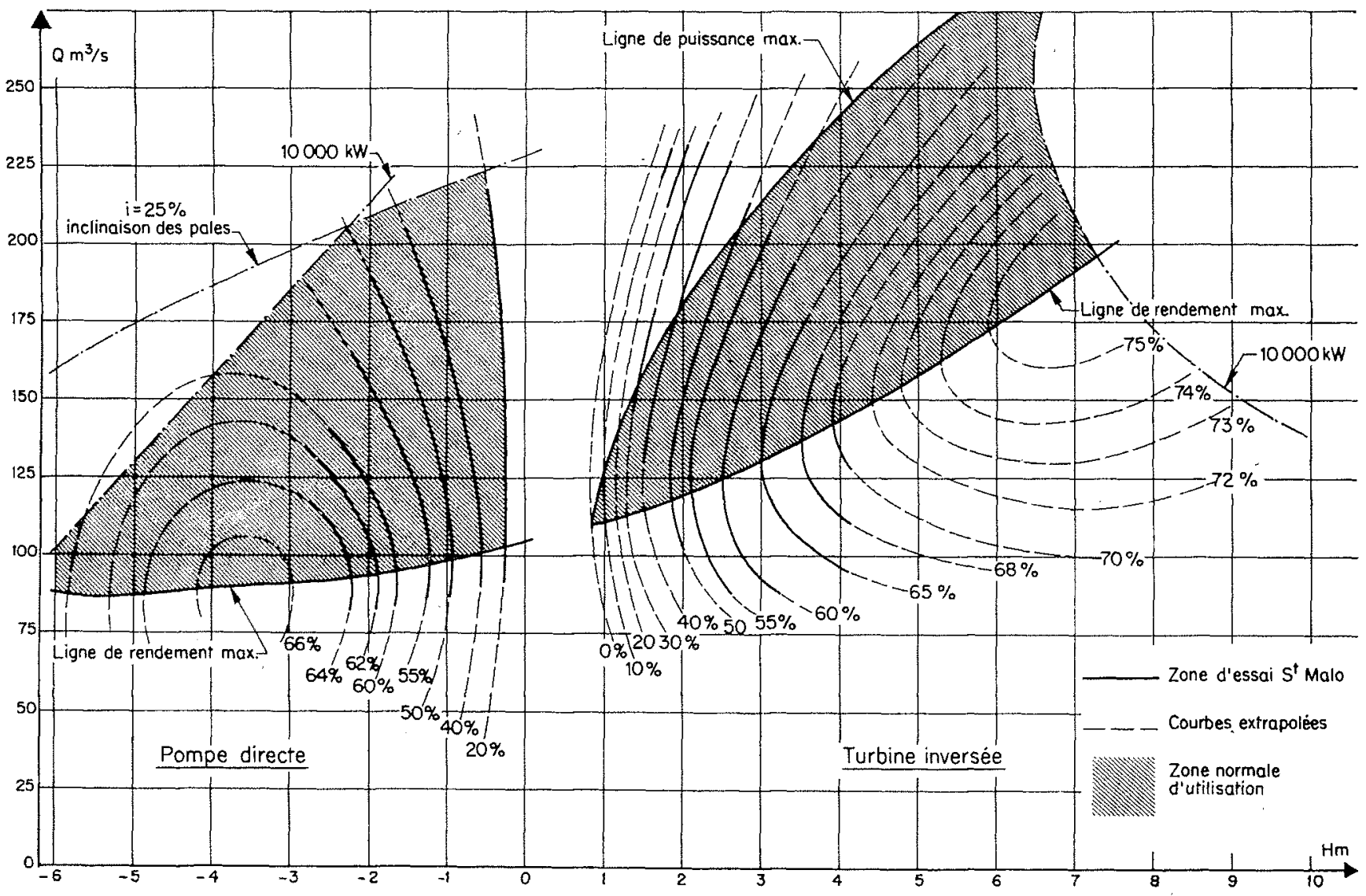

FIG. $5 a$

Saint-Malo. Diagramme topographique des rendements. 


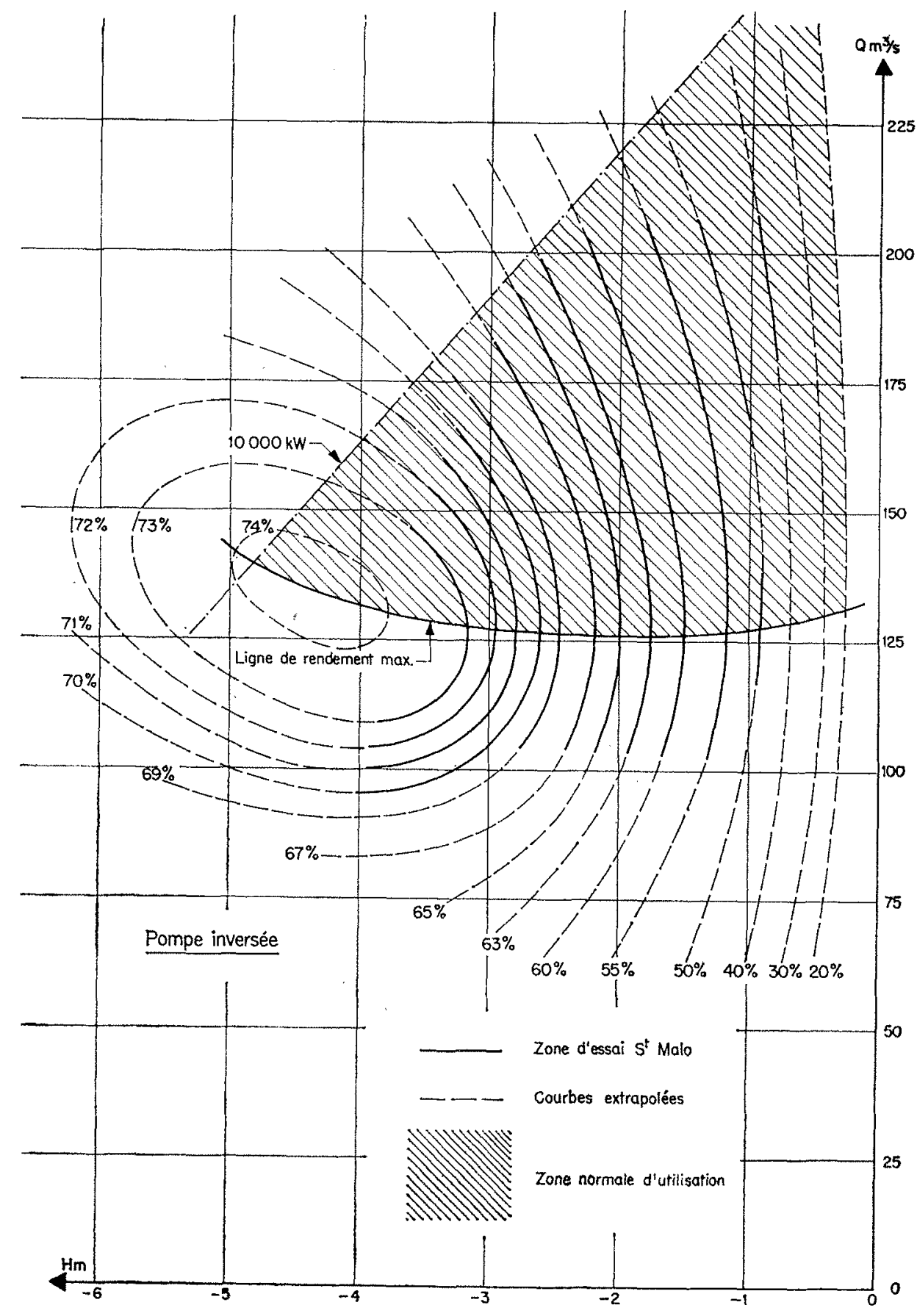

Saint-Malo.

Diagramme topographique du rendement.

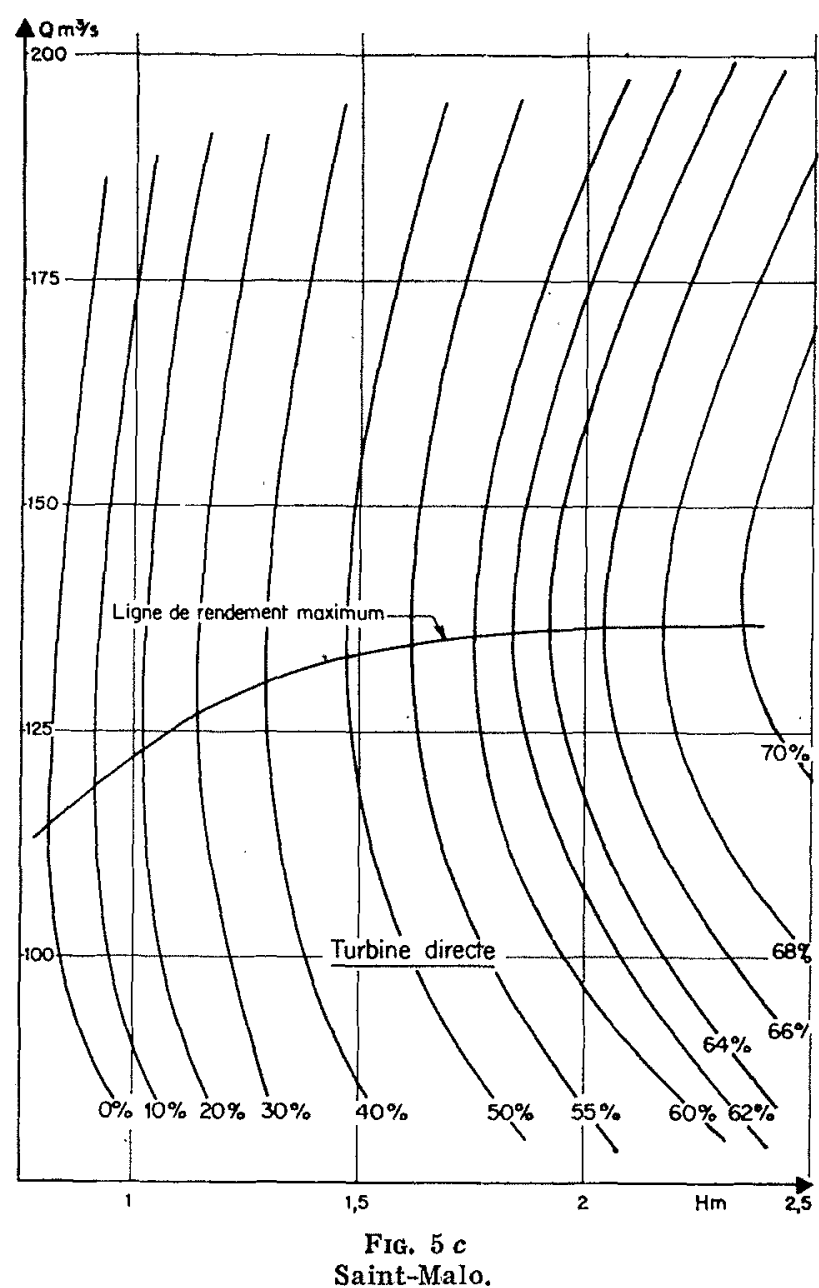

Diagramme topographique des rendements. 


\section{VI. - ESSAIS EN ORIFICE}

Certains cycles d'utilisation des groupes de la Rance comportant des fonctionnements en orifice, nous avons effectuć une série d'essais en orifice direct pour étudier le comportement de la machine dans ce type de fonctionnements.

Les mesures effectuées sur la machine étaient les suivantes :

\section{DéBIT.}

A la suite du dépouillement détaillé de tous les essais effectués en turbinage direct et pompage inversé, on a pu relier de façon suffisamment étroite le débit dans le pertuis d'entrée à la vitesse moyenne sur une horizontale située à $1,60 \mathrm{~m}$ au-dessus du radier, correspondant à la position de la perche supérieure du cadre lorsqu'il repose sur le radier. La mesure du débit a donc été faite en mesurant la vitesse sur cette seule horizontale.

\section{Chute.}

On a utilisé les disques de SER comme pour les précédents essais.

\section{VITESSE de Rotation.}

Enregistrement de la tension délivrée par une dynamo tachymétrique.

\section{Pression de part et d'autre de la rove.}

Cette mesure, comme celle de la vitesse de rotation, a pour but de déceler une éventuelle instabilité de l'écoulement.

Deux prises de pression ont été ménagées dans le plan horizontal de l'axe du groupe, à 1 mètre de part et d'autre du plan défini par les axes des pales. Ces prises étaient reliées d'une part à deux capteurs de pression absolue, d'autre part à un capteur différentiel enregistrant la différence de pression entre les deux prises.

\section{DÉplacement de L'arbre.}

Deux capteurs à mutuelle inductance ont permis de mesurer les déplacements de l'arbre au droit du palier de la turbine et au droit de la butée.

\section{PRESENTATION DES RESULTATS}

\section{a) Débit fit vitesse.}

Les graphiques des figures 6 et 7 montrent pour les inclinaisons de pales de 15,26 et $35^{\circ}$ l'évolution du débit et de la vitesse en fonction de l'ouverture $\alpha \mathrm{du}$ distributeur. On a éliminé le paramètre chute en ramenant tous les essais à deux mètres de chute, et tracé sur le même graphique les résultats relatifs à des essais sur modèle réduit.

On observe une allure générale concordante, avec toutefois un effet d'échelle plaçant le débit et la vitesse du prototype au-dessus des valeurs indiquées par le modèle. L'écart relativement important s'explique si l'on considère que, à l'effet d'échelle purement hydraulique on doit ajouter l'effet des pertes mécaniques qui sont relativement plus importantes sur le modèle que sur la machine industrielle, et dont on n'a pas tenu compte dans la présentation des résultats modèle.

Il est intéressant de noter que le débit maxi- mal, qui est logiquement recherché dans les fonctionnements en orifice, est obtenu quelle que soit l'inclinaison des pales pour une ouverture du distributeur voisine de 90 degrés.

\section{b) Fluctuations de pression.}

Les fluctuations de pression, dont quelques enregistrements sont reproduits figure 8, font apparaître deux phénomènes distincts :

- Aux faibles ouvertures du distributeur prend naissance une oscillation très régulière de fréquence voisine du tiers de la fréquence de la roue. Le fonctionnement de la machine, du fait de la déconjugaison importante, est mauvais du point de vue niveau de bruit de cavitation.

- Par contre, pour des ouvertures du distributeur supérieures à $70^{\circ}$, les oscillations disparaissent complètement, la dentelle apparaissant sur les enregistrements correspondant au passage des pales devant les prises de pression, qui en sont très proches. Le niveau de bruit devient 


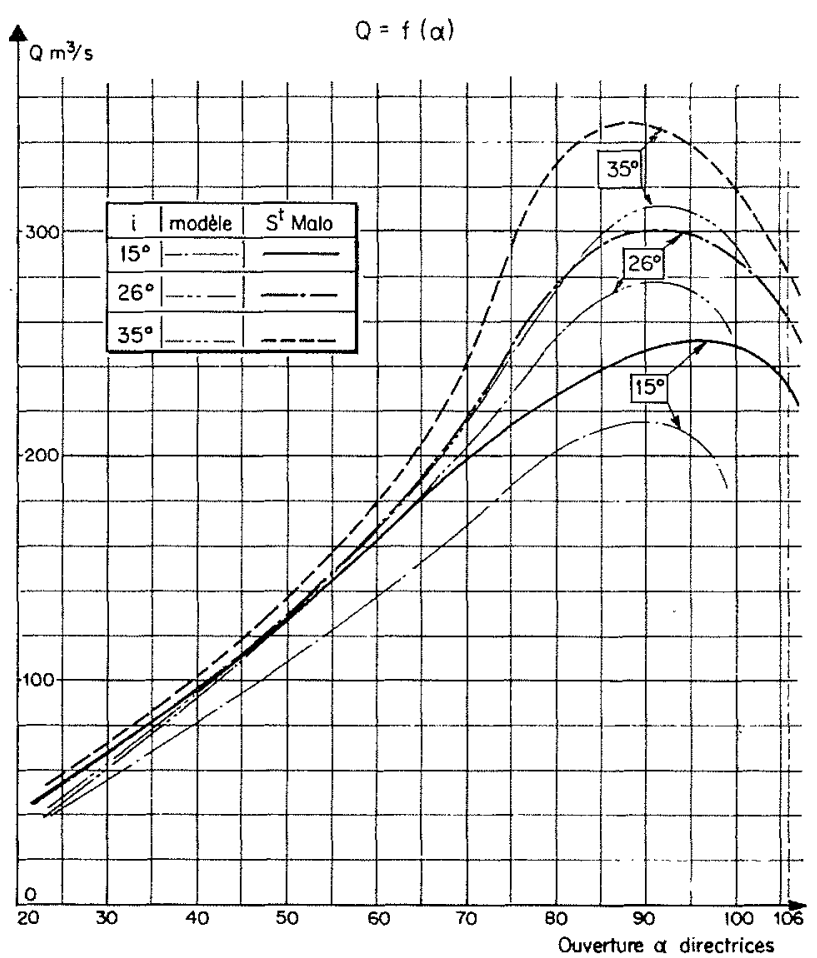

FIG. 6

Saint-Malo.

Orifice direct. $\mathrm{H}=2 \mathrm{~m}$.

$$
N=f(\alpha)
$$

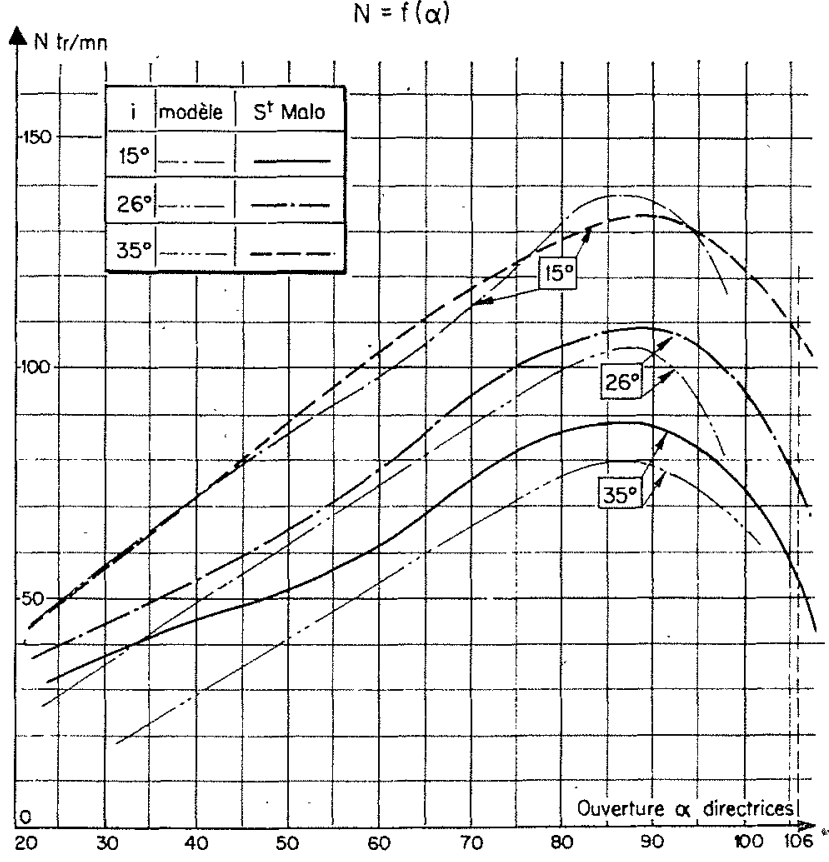

Fig. 7

Saint-Malo.

Orifice direct. $\mathrm{H}=2 \mathrm{~m}$. tout à fait normal et le fonctionnement de la machine satisfaisant.

c) Fluctuations de vitesse et déplacement de L'ARBRE.

On n'a relevé, à aucun régime, de fluctuations sensibles de vitesse. La figure 9 représente, en fonction de l'ouverture du distributeur, l'amplitude des déplacements du grain mobile de la butée $\mathrm{GMH}=f(x)$ et du palier turbine

$$
\mathrm{PTH}=f(\alpha) \text {. }
$$

On constate que les vibrations de l'arbre, comme les fluctuations de pression sont plus importantes aux faibles ouvertures du vannage. Les déplacements les plus importants apparaissent vers $40^{\circ}$ d'ouverture, leur amplitude atteint $2 / 10^{\circ}$ de millimètre. Au-dessus de $70^{\circ}$ d'ouverture l'amplitude demeure sensiblement égale à $1,2 / 10^{\circ}$, mais à aucun régime ces déplacements n’ont été jugés critiques.

d) Conclusion.

Il ressort des différents tests effectués sur la

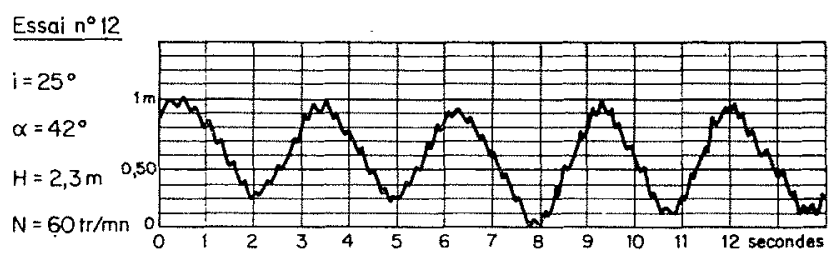

Essoi n $\pi^{\circ} 13$
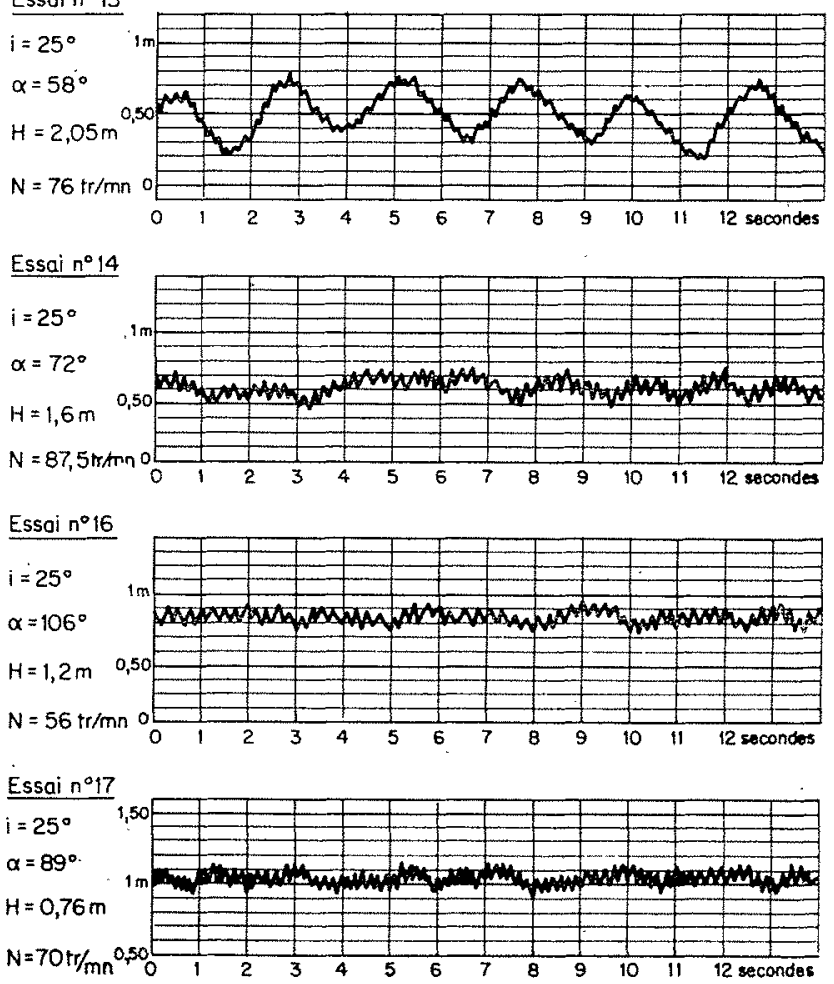

Fig. 8

Saint-Malo. Orifice direct.

Fluctuations de pression à l'aval de la rone. 


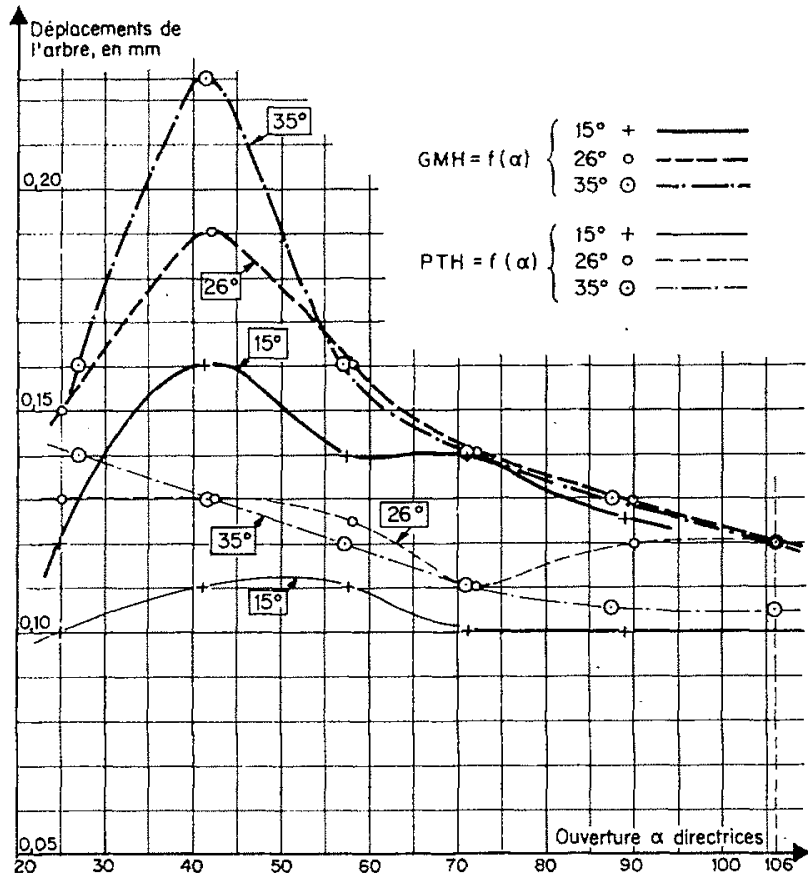

Fig. 9

Saint-Malo. Orifice direct. Déplacements de l'arbre. machine que, en ouvrant le distributeur à 90 degrés :

1. On se trouve très sensiblement, quelle que soit l'inclinaison des pales, au régime de débit maximal qui est recherché dans le fonctionnement en orifice.

2. Dans ces conditions les fluctuations de pression et les vibrations de l'arbre sont réduites au minimum.

Il faut cependant remarquer à nouveau pour terminer que les résultats présentés sont ramenés à la chute nette de deux mètres, alors que les essais ont été effectués à des chutes généralement inférieures. La vitesse maximale sous $2 \mathrm{~m}$ pour $15^{\circ}$ d'inclinaison des pales est de $132 \mathrm{tr} / \mathrm{mn}$ alors que la vitesse maximale réalisée aux essais est seulement de $120 \mathrm{tr} / \mathrm{mn}$. De même que le débit maximal sous $2 \mathrm{~m}$ et $35^{\circ}$ d'inclinaison est $340 \mathrm{~m}^{3} / \mathrm{s}$ alors que le débit maximal aux essais n'a été que de $265 \mathrm{~m}^{3} / \mathrm{s}$.

Cependant, bien que les conditions d'exploitation du groupe de Saint-Malo n'aient permis que d.es régimes limités, aucun indice défavorable ne s'oppose à la conclusion que le groupe se révélera capable d'assurer dans de bonnes conditions les fonctionnements en orifice direct qui lui seront demandés.

\section{NOTRE FRONTISPICE}

(Cf. page 2)

OHM (1787-1854)

Le physicien Ohm peut prendre rang parmi les savants dont les concepts ne furent pas appréciés à leur juste valeur par leurs contemporains. Lorsqu'en 1827 ses expériences, pour les. quelles il avait construit lui-mème les appareils nécessaires, le conduisirent à formuler la loi qui porte son nom, les résultats qu'il avait obtenus, et qui marquent pourtant une étape dans l'histoire de l'électricité, n'éveillèrent guère d'intérêt et furent accueillis sans enthousiasme.

Georges Simon Ohm, fils d'un serrurier, naquit à Erlangen (Allemagne) en 1787. Il fut successivement professeur de mathématiques et de physique au collège des Jésuites à Cologne (1817) puis à l'école de guerre de Berlin (1826), directeur de l'école puis a lecole de guerre de Berlin (1826), directeur de lécole polytechnique de Nuremberg (1833) et professeur de physique à I'Université de Munich (1849). Il mourut à Münich en 1854. On lui doit, entre autres ouvrages, la "Théorie mathématique des courants électriques $)$ (1827) traduite en français en 1860 , qui contenait les principales formules de propagation du courant dans un circuit ou dans une portion de circuit. Ohm $n^{\prime} y$ parlait pas de "resistance electrique", mais de "longueur réduite" représenté par lles, $c$ etant la conductibilite, donc linverse de ce qu'on nomme aujourd'hui la resistivité, $l$ et $s$ la largeur et la section du conducteur.

\section{OHM (1787-1854)}

This famous physicist was ane of those learned men whose ideas tere never fully appreciated during his lifetime. The famous law he formulated in 1827 and the results of the experiments he had carried out with entirely home-made apparatus were received with a singular lack of enthusiasm, despite the fact that they undeniably marked a major divance in electrical science. George Simon Ohm was born at Erlangen, in Germany, in 1787 A iocksmith's son, he became a Professor of Mathematics and Physics at the Jesuit College in Berlin in 1817 and then at the Berlin War College in 1826. he was subsequently a Director of the Nuremberg Polytechnic School subsequently a Director of Physics at Munich Univensity (1849). His death occurred of Munich in 1854 .

Perhaps the most famous of his works was the "Matinematical Theory of Electric Currents" (published in 1827 and translated into French in 1860), which contains all the principal formulae for the propagation of an electric current in a whole or partial for the propagation of an electric current in a whole or partia circuit. Ohm did not refer to electric resistance" as such the to "reduced length, which he defined as being the length of the conductor divided by the product of conductivity times con ductor cross-sectional area', 'conductivity' being the reciprocal of what we nowadays call 'resistivity'. 